\title{
Multimodal Vessel Visualization of Mouse Aorta PET/CT Scans
}

\author{
Timo Ropinski, Member, IEEE, Sven Hermann, Rainer Reich, Michael Schäfers, and Klaus Hinrichs, Member, IEEE
}

\begin{abstract}
In this paper, we present a visualization system for the visual analysis of PET/CT scans of aortic arches of mice. The system has been designed in close collaboration between researchers from the areas of visualization and molecular imaging with the objective to get deeper insights into the structural and molecular processes which take place during plaque development. Understanding the development of plaques might lead to a better and earlier diagnosis of cardiovascular diseases, which are still the main cause of death in the western world. After motivating our approach, we will briefly describe the multimodal data acquisition process before explaining the visualization techniques used. The main goal is to develop a system which supports visual comparison of the data of different species. Therefore, we have chosen a linked multi-view approach, which amongst others integrates a specialized straightened multipath curved planar reformation and a multimodal vessel flattening technique. We have applied the visualization concepts to multiple data sets, and we will present the results of this investigation.
\end{abstract}

Index Terms-Vessel visualization, plaque growth, multipath CPR, vessel flattening.

\section{INTRODUCTION}

According to the 2008 report of the European Heart Network, cardiovascular events are responsible for over $50 \%$ of all deaths in Europe with over 2 million people dying every year. Although preventive measures on the population level and curative medicine have contributed to the continuing decline of cardiovascular mortality over recent years, early identification of the individual subject at risk of cardiovascular complications remains a great challenge. Most of the vascular pathologies and the majority of cardiovascular events arise from atherosclerosis which is characterized by typical inflammatory degenerative processes in the vessel wall. The main pathologic feature of atherosclerosis is the atherosclerotic lesion (plaque). It develops during a series of highly specific cellular and molecular inflammatory processes in the vessel wall and leads primarily to a constriction of the vessel (stenosis) $[8,23]$. The vascular inflammation is characterized and driven by infiltration of cells into the vessel wall in conjunction with a concomitant incorporation of lipids and deposition of calcium. The individual prognosis of the plaque and the patient with respect to plaque rupture and the subsequent life-threatening events myocardial infarction or stroke is determined by the structural, functional and molecular characteristics of the plaque. An integrated approach combining morphological, functional and molecular imaging modalities to multi-parametrically characterize atherosclerotic lesions should evolve as a powerful clinical tool to predict and prevent future cardiovascular events. This goal can only be achieved by incorporating a multitude of parameters derived from different modalities to gain a profound understanding of the development of atherosclerosis. In this context, the search for specific molecular markers, which allow to image the different states of atherosclerosis development, is an ongoing research direction in molecular imaging. To validate the parameters such as inflammation, local enzyme activity, vessel diameter or calcium deposits, which are acquired through imaging by using new molecular markers, mouse models of atherosclerotic lesions are established. Thus the validation of new markers and imaging technologies as well as the understanding of the process of plaque development

- Timo Ropinski, Rainer Reich and Klaus Hinrichs are with the Visualization and Computer Graphics Research Group (VisCG), University of Münster, E-mail: $\{$ ropinski|r_reic02|khh\}@math.uni-muenster.de.

- Sven Hermann is with the University Hospital of Münster, E-mail: shermann@uni-muenster.de.

- Michael Schäfers is with the European Institute of Molecular Imaging (EIMI),E-mail: schafmi@uni-muenster.de.

Manuscript received 31 March 2009; accepted 27 July 2009; posted online 11 October 2009; mailed on 5 October 2009.

For information on obtaining reprints of this article, please send email to:tvcg@computer.org . within the mouse model is the first step towards classifying the state of atherosclerosis in human beings in order to support a better and earlier diagnosis of cardiovascular diseases.

While medical workstations are established for clinical practice, most medical research oriented tasks can only, if at all, be performed by combining different software tools for medical imaging, which have their strengths and weaknesses in different aspects, e. g., registration, visualization or quantification. However, most often research questions cannot be answered without implementing new techniques which have been adapted to the current needs. Furthermore, exchanging data between different software tools is error prone and results in a lengthy analysis process. As a consequence, besides the challenges behind the development of the innovative imaging technologies, new visualization systems for the interactive analysis of the resulting multiparametric imaging data are needed.

In this paper, we describe a visualization system, which has been developed collaboratively by researchers from visualization as well as molecular imaging. The goal of the system is to optimally support the work flow when exploring combined vessel imaging data from high resolution small animal positron emission tomography (PET) and computed tomography (CT), where the PET data shows inflammatory plaque activity and the CT data provides information about degree of stenosis, calcification, wall thickness and such. With the proposed system, it becomes possible to intuitively and efficiently use multimodal imaging data to clinically characterize the complex scenario of atherosclerotic plaques in the aortic arches of different mouse models. The system has been designed with the objective to facilitate the spatial understanding of imaged plaque regions and to support a quantitative in depth analysis. By exploiting comparative visualization strategies, individual data sets can be compared to control groups in order to be able to compare mouse models or mice fed with different diets.

\section{Related Work}

Although 2D slice views are frequently used in medical visualization, they are unsuitable for visualizing longitudinal structures such as vessels. There are mainly two alternative approaches for the visual exploration of vessel structures: $3 \mathrm{D}$ visualizations and different variants of planar projections. 3D visualizations often exploit modelbased approaches, which abstract the geometry of the vessels to be visualized $[4,21]$, but more recently also model-free visualization techniques have been proposed, which reduce the degree of abstraction [25]. While 3D approaches have the advantage that they reflect the overall structure of the vessel very well, they have limitations when visualizing a vessel's interior. Therefore, different projection based visualization techniques have been exploited. Kanitsar et al. have presented techniques for generating curved planar reformations (CPRs), where longitudinal cross sections are generated to show parts of the vessel's lumen as well as the surrounding tissue in a curved plane [13]. 
In their paper they discuss three CPR variants: projected, stretched and straightened. Since the generated longitudinal cross-sections do not cover the whole vessel, they present Thick CPR, where different compositing schemes are exploited to accumulate the data for the entire lumen. Furthermore, they propose an image-based algorithm for combining multiple CPRs within one image. In more recent publications, the same authors present further CPR improvements $[14,15]$. By applying an untangling process, they are able to reduce the occlusion of multiple overlapping vessels in a multipath CPR. Won et al. have proposed an alternative approach to provide an uncluttered view to the abdominal aorta and its branches [32]. By combining the abstraction of a vessel's geometry through convex primitives with an appropriate placement algorithm, they were able to generate one uncluttered image containing all relevant information. Besides these image-based approaches for integrating multiple branches into one visualization, also center line reformation techniques have been proposed. He et al. refine the center line by using a multi-scale approach, before they flatten the tree semiautomatically by using a so-called medial axis reformation [10]. More recently, Lee et al. did also focus on the problem of non-planarity of vascular trees [20]. They reduce visualization artifacts introduced by non-planar vascular tree alignment by using a high-level description of the medial axis. This description is exploited in order to achieve a reformation process which embeds the vascular tree into the image plane.

While CPR techniques are frequently employed in the medical diagnosis process, they have the drawback that not the entire vessel lumen can be visualized. Therefore, Kanitsar et al. use a helical sampling pattern to show the vessel lumen [15]. Besides that, other flattening strategies have been applied to vessels. Zhu et al. present a conformal flattening technique for vessel systems, which is based on a discrete representation of the Laplace-Beltrami operator $[34,36]$. In a later publication by the same authors a technique for achieving an area-preserving flattening of vessels is described, which is based on the mass transport theory [35]. Not only vessel visualization techniques present complex structures by a projection into a plane, similar approaches are also exploited in the area of virtual endoscopy. For instance, conformal colon flattening is used to project the surface of the colon into a plane to support improved inspection [11]. More recently, Williams et al. have presented an approach for virtual endoscopy by utilizing CPR in combination with DVR [30]. They use volume rendering for hollow regions, which can be combined with one of the three standard CPR techniques applied to the surrounding tissue. To improve the integration of contextual structures into CPR visualizations, Straka et al. present a combination of DVR and CPR techniques [27]. By integrating a CPR into a DVR rendering, they are able to generate an easy to comprehend focus and context visualization of vessel structures.

Additionally, techniques have been proposed in the volume rendering community to support data deformations. Laidlaw has described a ray-based algorithm, which casts rays along deformed paths in order to generate a variety of deformations [18]. The approach of Brunet et al. focuses more on the exploitation of the capabilities of graphics hardware [3]. By using slicing in combination with their deformation function, the desired deformations can be applied interactively with their proposed deformation widgets.

Besides these more technical papers, also more general system papers can be found in the literature. For instance, Wink et al. propose a vessel analysis system which is based on a 3D visualization of angiography data sets [31]. By interactively setting the start- and endpoints of a vessel, a center line computation is initiated and the minimum diameter as well as a surface representation of the vessel is computed. Hahn et al. describe the general concepts underlying a vessel visualization pipeline [9], starting with the center line detection and processing before focusing on the actual visualization issues. Similar to the approach described in this paper, the VascuVision system also exploits multiple linked views in order to support the visual analysis of vessels [7]. The system can be used to interactively extract the center lines of vessels before visualizing them in 3D as well as by using CPR. Furthermore, the vessel diameter and cross cuts along the vessel can be

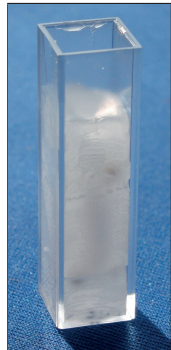

(a)

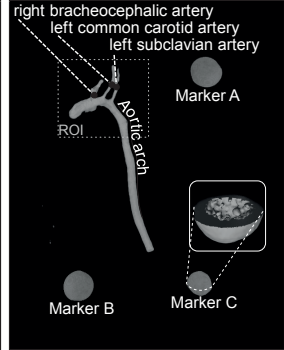

(b)

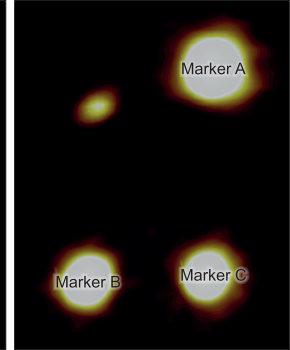

(c)
Fig. 1. The preparation showing a cast of an aortic arch of a mouse embedded in Paraffin (a), and resulting renderings of the CT (b) and PET (c) scans of the Paraffin block. As it can be seen in (b) and (c), three markers have been added to support the registration of the modalities. The inset in (b) shows a cut through a marker in the CT data set.

displayed. However, multipath CPR for comparative visualization and multimodal techniques have not been integrated into the VascuVision system. A 3D visualization approach based on harmonic skeletons for analyzing stenosis has been presented by Yang et al. [33]. Besides these techniques specialized on vessel visualization, also more general multiview systems for medical visualization have been proposed. For instance, Kohlmann et al. have described an automatic approach for adjusting 3D rendering parameters by selecting structures of interest in a coupled 2D view [16]. Additionally, many publications targeting multimodal visualization exist. For instance, Beyer et al. propose an application for neurosurgical planning, which allows to visualize anatomical and functional data in an integrated manner by exploiting multi-volume rendering based on a brick data structure [1]. More recently, Rieder et al. have proposed a multimodal approach for planning head tumor treatment by also considering anatomical and functional data of the brain [22]. In contrast to these approaches, multimodal vessel visualization techniques need to convey both the overall vessel structure as well as the vessel's interior.

\section{Data Acquisition and Registration}

To gain knowledge about the process of plaque development, ex-vivo preparations of the aortic arch of different mouse models have been generated. Each preparation consists of a cuvette filled with Paraffin, into which one aortic arch filled with Microfil contrast agent (Flow Tech Inc, Massachusetts) has been embedded (see Figure 1 (a)). Besides the aortic arch the three outgoing vessels, i. e., the right bracheocephalic artery, the left common carotid artery and the left subclavian artery, are preserved in the preparations. The Microfil, which has been injected before dissection, serves as contrast agent and generates a cast of the vessels. We have chosen the density of the Microfil such that the intensity ranges of the Microfil, the vessel wall and the Paraffin do not overlap. Avoidance of an overlap in combination with the standardized CT scanning protocol allows us to distinguish the lumen, the vessel wall as well as the outer regions based on intensity ranges. This is essential for all visualization techniques described in Section 4. To be able to examine besides the morphological structures also the current state of plaque growth, radioactive PET tracers have been injected prior to dissection. Before discussing the visualization techniques exploited for the visual analysis, in the remainder of this section we will briefly describe the data acquisition and the necessary data processing from a technical point of view. More details regarding the mouse models and the PET markers can be found in Section 5.

A PET as well as a CT rendering of the aortic arch embedded in Paraffin is shown in Figure 1 (b) and (c). While the aorta itself has a diameter of about $0.7-1.4 \mathrm{~mm}$, the outgoing vessels are even smaller. Therefore, high resolution imaging devices are needed for the acquisition. We use the very high resolution QuadHidac scanner for acquiring the PET modality and a Siemens Inveon small animal CT scanner for the CT acquisition. The QuadHidac scanner has a resolution of $0.7 \mathrm{~mm}$ (full width half maximum with resolution recovery) and produces 16 


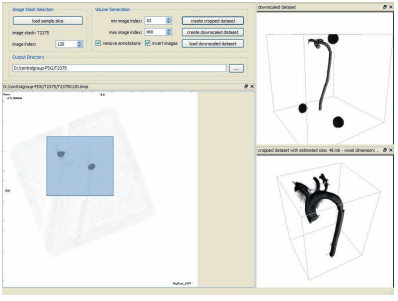

(a)

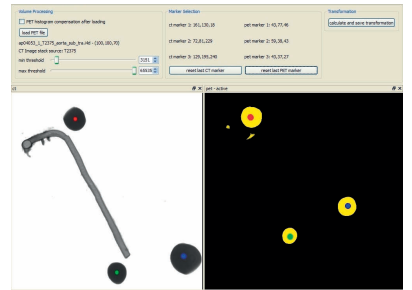

(b)
Fig. 2. Screenshots of the interactive cropping and registration dialog. By selecting the region of interest, the user can generate a cropped data set (a). By double clicking on a marker, the user initiates a region growing process, which is used to determine the center of the markers, which are depicted by the differently colored circles (b).

bit data sets consisting of $160 \times 90 \times 70$ voxels, whereas the CT scanner has a resolution of $15 \mu \mathrm{m}$ and generates 8 bit data sets consisting of $1024 \times 1024 \times 1023$ voxels. Since this amount of data is too large to handle for most consumer graphics boards and, as it can be seen in Figure 1 (b), only rather small areas of the volume are occupied by the aortic arch, we provide an interactive dialog for cropping the data sets (see Figure 2 (a)). Thus, only the region of interest is contained in the data to be processed, and all data sets fit into graphics memory.

Since the two modalities have been acquired with different scanners, a registration has to be performed. While there are sophisticated algorithms for registering two similar data sets, for instance two CT data sets, the problem is a little more complex in the described case. As it can be seen in Figure 1 (c), in the PET data set no matching morphological structures can be exploited as landmarks for the registration. Thus, interactively selecting points of interest within the data sets is difficult. Therefore we exploit three spherical ceramic markers which have been inserted into the Paraffin block. To ensure marker visibility in both modalities (recall Figure 1 (b) and (c)), the radiopaque markers were also labeled with the radioisotope F-18-Fluoride.

The actual registration is done in another dialog, which allows to interactively select the markers, before detecting the center of each marker in both PET and CT and computing the desired transformation matrix $T$ (see Figure 2 (b)). Based on the computed centers, we have implemented a least-squares solver for the multimodal registration. While the theory behind this registration process is well understood, we had to deal with some practical problems. To detect the markers within the data set, we have implemented a threshold-based region growing approach [12] for which the seed points can be placed manually with a single mouse click. One problem with the region growing approach is the fact that not all markers had a uniform intensity inside. While the radioactive PET tracer was generally absorbed very well, in many cases the markers showed an intrinsic inhomogeneous density in the CT data set (see inset in Figure 1 (b)). When assuming that the markers approximate a spherical shape, the mean of all voxel positions belonging to the marker, i. e., the result of the region growing process, would be a good approximation of the center. However, since in our case the intensity is distributed non-uniformly towards the center of the marker, and thus not all marker voxels can be detected during a single region growing pass, we had to adapt this approach. In the first step, we determine an intermediate center by computing the mean of all voxel positions belonging to the filled region. Then, in a second step, we use this center as a seed point for another boundary-based region growing process, which fills the empty regions inside the marker. This is possible since the surface is the most dense part of the marker. Then we could compute the new center for the thus determined marker region by computing the mean of all marker voxel positions. While this is a rather simple approach, the validation of the registration presented in Section 5 has shown that it works well in practice. After determining all three center points in both modalities in the same order, the least squares solver can be used for computing the registration transformation $T$.
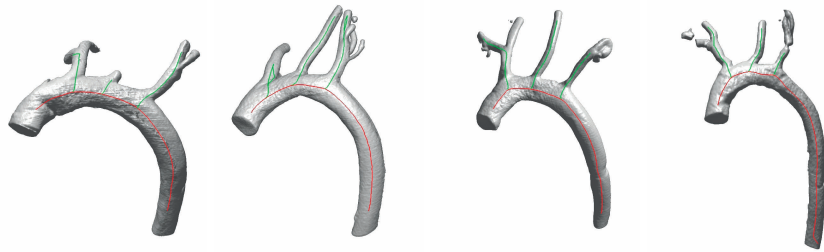

Fig. 3. When generating comparative visualizations, dealing with biological variance is challenging. The images show CT renderings of the lumen of the aortic arch of four different mice. The colored lines represent the computed center lines for the aortic arch (red) and the outgoing vessels (green).

\section{Comparative Multimodal Visualization}

As mentioned in Section 1, the goal of this work is to identify and visually analyze the different stages of plaque growth in the aortic arch of the mouse as a reference model. Before presenting the visualization approaches exploited to reach this goal, we briefly summarize our design decisions, which have been made based on the four following affordances.

Spatial comprehension. As required by most approaches in the area of scientific visualization, spatial comprehension is a key criterion. While $2 \mathrm{D}$ slices are a good choice for many daily routine diagnostic tasks, they are insufficient for inspecting longitudinal structures such as vessels, which may intersect multiple adjacent slices in an awkward manner. In general, 3D displays are good for gaining an overview of a 3D space and for understanding 3D shapes [28]. Accordingly, 3D visualizations of vessels result in an improved spatial comprehension (see for instance Figure 1 (b), Figure 3 or Figure 5). However, they suffer from other drawbacks such as occlusion or, in our case more prominent, the disadvantage of not being able to appropriately visualize the interior of the vessels.

Multimodal/multi-parametric visualization. To support the inspection of structural, functional and molecular characteristics of a plaque, the inspection has to be done based on the multimodal PET/CT data acquired and registered as described in Section 3. Furthermore, the system needs to be able to integrate further variables derived from the data set such as wall thickness and lumen diameter.

Comparative visualization. The development of atherosclerosis is a well-defined chronic process in different animal models. These models are typically based on gene knock-outs in mice, which lead to variable dynamics and manifestation of the disease. Therefore, the intraindividual follow-up in a single mouse over time as well as the interindividual comparison between different mouse models or with control groups is of interest and comparative visualization approaches are required. The main challenge in the area of comparative visualization is variability. Due to biological variance and preparation artifacts, no direct mapping between different data sets is possible (see Figure 3). Thus it has to be ensured that a mapping between more or less differently shaped structures can be determined. Furthermore, this visualization should support an in-detail inspection by allowing to establish precise relationships between different regions in the same data set as well as different data sets. Since Springmeyer et al. did observe that these relationships can be best established in 2D [26], we have decided to use a 2D comparative visualization.

Quantification. Finally, all visualized parameters must be quantifiable. When comparing different mouse models, not only relative conclusions have to be drawn, but also quantitative ones. Thus, parameters such as wall thickness, vessel lumen and regional uptake of molecular markers need to be displayed numerically.

Obviously the ultimate goal is to meet all the affordances described above. However, there exists a strong dependency between the affordance of spatial comprehension and the affordance of comparative visualization. To deal with the variability of the data sets, a normalization is required in order to support a comparative visualization, i. e., a mapping must be found which maps all data sets into a unified space. 


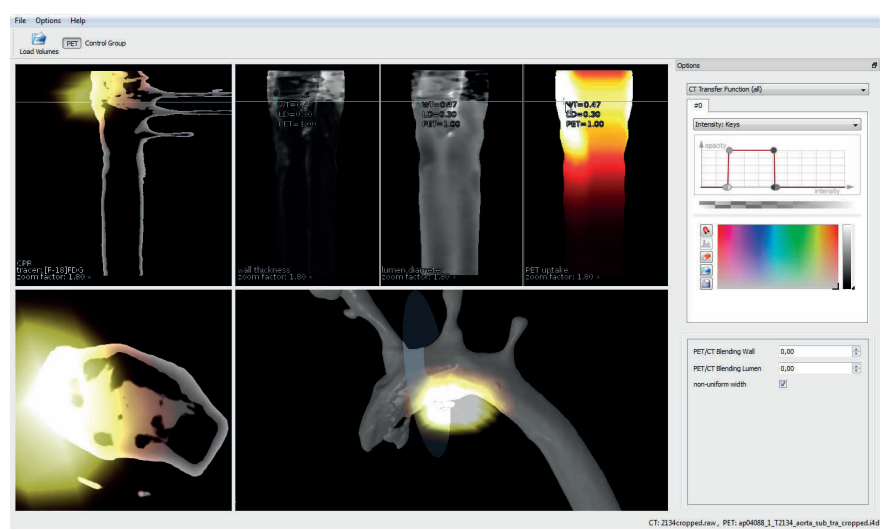

Fig. 4. Multiple linked views are employed to allow a comparative visualization of aortic arches of mice while still supporting spatial comprehension. From upper left to lower right: A multipath straightened CPR, flattening views, showing wall thickness, lumen diameter and PET uptake. The 2D cross section allows in-detail inspection, and the 3D view supports spatial comprehension by providing an overview.

Unfortunately, this mapping will result in distortion to some degree and thus affects spatial comprehension. Therefore, we decided to exploit a linked multi-view approach in order to support both spatial comprehension and comparative visualization at the same time. However, simply linking a 3D visualization with a normalized representation does not help much. For instance, in our case, due to the applied normalization, the flattening exhibits a vertical non-linearity of the different segments of the aortic arch. Additionally, while the 3D view of the lumen reflects the diameter of the vessel, this is not the case in the normalized visualization, which further affects spatial comprehension. Thus, especially when the user changes the viewing parameters in the $3 \mathrm{D}$ view, we experienced a high cognitive effort when trying to mentally register the linked views. Therefore we have chosen to integrate an intermediate visualization to support the mental linking of the 3D visualization with the normalized visualization.

Since mental registration comprises a pattern matching, where unique features in one view are matched with features in the other view [28], we need to incorporate unique features within the available views. For the aortic arch these features are clearly given by the right bracheocephalic artery, the left common carotid artery and the left subclavian artery (recall Figure 1 (b)). Thus an already normalized visualization, which still shows the overall structure of the aortic arch with the outgoing vessels, could be a successful link between the 2D and the $3 \mathrm{D}$ visualization. We have decided to use a modified straightened CPR technique together with vertical normalization to achieve this goal. While this reduces occlusion, we are able to depict the undistorted diameter of the vessel as well as the positions of the arteries. By introducing this intermediate visualization, the abstraction of the 3D view towards the normalized visualization is divided into two significantly smaller stages of abstraction. Figure 4 shows the linked multi-view system containing the discussed views plus a $2 \mathrm{D}$ cross sectional view. In the following subsections we will describe the techniques used to generate the views as well as the realized interaction metaphors.

\subsection{D Visualization}

For the 3D view of the vessels, we have implemented a GPU-based ray-caster based on the rasterization of the volume's proxy geometry to obtain entry- and exit-points [17]. To visualize multimodal data sets, we had to apply two modifications to the original algorithm. First, we had to ensure that the registration transformation $T$ is applied, and second, we had to develop a novel compositing scheme, which allows to combine DVR compositing as used for CT with MIP compositing as used for PET.

The application of $T$ during the visualization process is necessary since we have decided not to apply resampling. This proceeding is
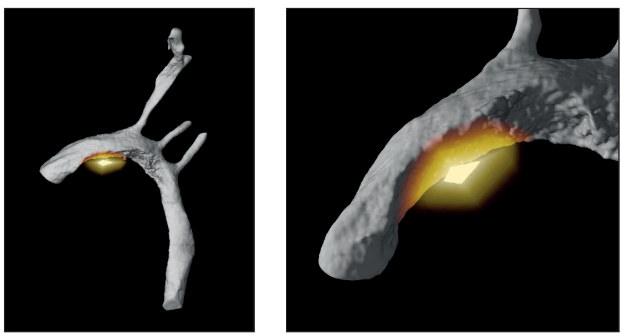

Fig. 5. Two different views of our modified GPU-based ray-caster, which allows to generate multimodal renderings at interactive frame rates by exploiting a combined DVR/MIP compositing scheme. Both images show the lumen of the vessel and the hot region located in the vessel wall. The vessel wall itself is excluded from the visualization in order to reveal the narrowing of the lumen.

beneficial since we have differences in resolution of a factor of over 6 between the two modalities (recall Section 3). Thus, resampling the higher resolution CT data set would result in a loss of information, while resampling the lower-resolution PET data set would lead to unnecessary high memory consumption. Applying $T$ during the rasterization of the proxy geometry helps to avoid these problems and additionally conserves the original data sets. Now that we have two registered proxy geometries, they can be rendered, and the actual raycasting can be performed. We have chosen to follow an approach proposed by Brecheisen et al. [2], which exploits depth peeling in order to generate the successive pairs of entry- and exit-positions for the two modalities. The only modification is affecting the compositing as done during the ray marching. Just blending the result of the DVR CT rendering and the MIP PET rendering would affect the spatial comprehension, since high PET intensities lying behind the lumen wall, which is displayed opaque in the DVR, would be visible. To get a correct occlusion relation, during the MIP generation we only consider those PET samples which lie in front of the lumen wall.

By using this modified GPU-based ray-caster, we are able to generate multimodal renderings at interactive frame rates such as those shown in Figure 5. Based on the known intensity ranges of the wall and the lumen, we have chosen the transfer function in a way that only the lumen of the vessel is visible. By visually suppressing the vessel wall, we ensure that the PET uptake, which is entirely located inside the wall, is completely visible. The color scales have been chosen to match those widely used in clinical practice.

\subsection{Straightened Multipath CPR}

As mentioned above, one of the goals of our modified CPR approach is to support spatial comprehension by providing an intermediate visualization, which already incorporates some normalization. Therefore we have adapted the straightened CPR approach, which is a specialized form of a CPR, where the center line of the vessel is straightened before projecting it onto the image plane [13]. To deal with the bad perceptual qualities of straightened $\mathrm{CPR}$, we have further integrated the branching vessels as visual landmarks. Although due to the applied compositing, even the Thick CPR variant cannot capture the entire information for a vessel's interior [13], this does not pose a problem in our case, since we additionally exploit a flattening technique (see Subsection 4.4) for detailed inspection of the vessel's interior.

\subsubsection{Center Line Computation}

Kanitsar et al. state that the most important prerequisite for CPR visualization is an appropriate estimation of the center line of a vessel [14] Among different tested algorithms, the curve skeleton approach $[6,5]$ led to the best results for all those available data sets which did not suffer from too serious preparation artifacts, as for instance a split aortic arch. By using this algorithm, we compute the center line of the segment containing the lumen and the vessel wall, instead of just the lumen, in order to get a less noisy center line for sick mice suffering from a high degree of stenosis. 


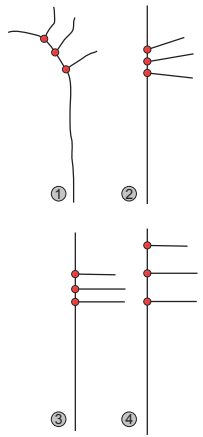

(a)

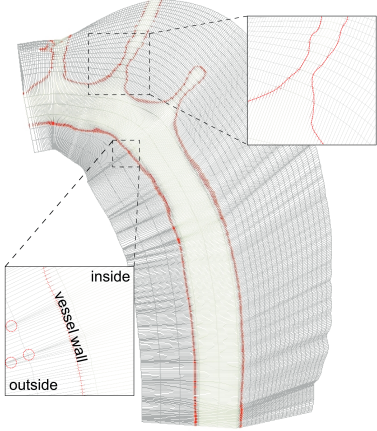

(b)
Fig. 6 . To be able to provide a comparative visualization of different data sets, we have to normalize the vessel's geometry. The four conceptual stages of the normalization procedure are, as depicted in (a): (1) the center line, (2) the straightened center line, (3) the straightened center line with $90^{\circ}$ bifurcation angles, and (4) the straightened center line with normalized segments. (b) shows a polygonal mesh derived from the center line, which represents the lines of interest $l\left(p_{i}\right)$ for all vessel segments; the upper inset shows the continuous $l\left(p_{i}\right)$ at the second bifurcation, while the lower inset shows that intersections (red circles) between adjacent cross sections only occur outside the vessel.

Since a typical curve skeleton is given by multiple segments, further processing is required to transform the skeleton into a center line, as those shown in Figure 3. To classify and connect the segments, we select a curve skeleton segment, which belongs to the thickest part of the vessel, i. e., the aortic arch. Starting from that segment, we traverse the adjacent segments and connect them to generate the center line for the arch. Then we traverse this center line and use the same connection technique at the bifurcations to identify outgoing segments. After these have been identified, we select the three outgoing segments having maximum length as the desired outgoing vessels. The result of this processing is a classified center line as shown in Figure 3, where the aortic arch is depicted in red and the outgoing vessels are depicted in green. While in general the used curve skeleton algorithm produced reliable results for the tested data sets, a problem occurred at the bifurcations of the center lines. As it can be seen in Figure 3, the bifurcations are rather represented as curves, which do not capture the actual angle of bifurcation as lying inside the arch. If we would use the bifurcation computed by the curve skeleton algorithm, the resulting CPR would suffer from distortion as well as overlap within the arch. Since this is the actual region of interest, we have modified the bifurcations represented by the centerline. At each bifurcation, we choose the point $p_{o}$ on the center line of the outgoing vessel, which is closest to the center line of the aortic arch, while the vessel diameter at that position still equals the diameter of an outgoing vessel. We compute the vector $v_{0}=\left|p_{a_{0}}-p_{o}\right|$, where $p_{a_{0}}$ represents the computed bifurcation point. To find the desired bifurcation point, we traverse the arch's center line and choose successive $p_{a_{i}}$ until the equation $v_{i} \cdot t_{i} \approx 0$ is satisfied, where $t_{i}$ represents the tangent of the center line at location $p_{a_{i}}$. Then we eliminate the center line segment between $p_{o}$ and $p_{a_{0}}$ and introduce a new straight segment between $p_{o}$ and $p_{a_{i}}$.

\subsubsection{Computing Reformation Parameters}

Based on the computed center lines, we can introduce the desired normalization by still incorporating distinctive features. Thus, we have to ensure that the aortic arch as well as the outgoing vessels are transformed to the same position for different data sets by achieving smooth transitions at the bifurcations. The conceptual stages of this normalization process are displayed in Figure 6 (a). The goal is to create a representation in which all vessels are straightened, the outgoing vessels are modified to lie in the same plane, i. e., the viewing plane, and the angle of bifurcations is adapted to be $90^{\circ}$ within this plane. To achieve complete normalization of the center lines, we have to stretch or skew the different segments in such a way that the center line segments between the right bracheocephalic artery and the left common carotid artery as well as between the left common carotid artery and the left subclavian artery (recall Figure 1 (b)) have the same lengths for all data sets.

We have implemented a GPU-based geometry morphing approach to enable the straightening as well as the described normalization. For generating the CPR, we first need to determine the lines of interest $l\left(p_{i}\right)$. These are computed for all $p_{i}$ lying on the main arch based on the three bifurcations by applying a simple interpolation approach. As input for the interpolation, we set the $l\left(p_{i}\right)$ at the $p_{a_{i}}$ to $v_{i}$. Thus we ensure that the line of interest equals the center line of the outgoing vessels. Now that we have computed the lines of interest for all determined $p_{a_{i}}$ on the arch, we can use linear interpolation to compute the lines of interest for all $p_{i}$ on the center line of the arch. As the $l\left(p_{i}\right)$ for all $p_{i}$ on the outgoing vessels, we have chosen $t_{i}$. We can use the thus computed $l\left(p_{i}\right)$ as a basis for a mesh as shown in Figure 6 (b). To generate geometry for the parts of the mesh, where no line of interest is known, we generate the missing lines of interest by interpolating resp. extrapolating the determined $l\left(p_{i}\right)$. To avoid a bending of the projection planes intersecting the outgoing vessels, the $l\left(p_{i}\right)$ are set constant across the maximum diameter of the outgoing vessels. As it can be seen in the upper inset in Figure 6 (b), the described approach results in smooth transitions at the bifurcations independent of the zoom level. Furthermore, the lower inset shows that the patches used to build up the mesh do not overlap for adjacent cross sections. Even for the regions with the highest curvature, overlapping as denoted by the circles surrounding the intersection points occurs only outside the vessel and thus does not affect the visualization.

\subsubsection{Rendering}

The actual straightening and normalization can be simply applied when rendering a proxy-mesh having the same grid structure as the mesh shown in Figure 6 (b). Since the vertex positions in Figure 6 (b) are given in volume coordinates, we can simply assign these coordinates as texture coordinates to the vertices of the proxy-mesh. During rendering we use these texture coordinates to perform a texture lookup in the volume texture, which represents the current data set. By choosing a rectangular silhouette for the proxy-mesh and vertically stretching resp. skewing the areas representing the segments between the outgoing vessels, both straightening and normalization become possible. To show PET and CT at the same time, we allow to interactively blend the CPR of the PET data set, which is generated by exploiting the MIP paradigm, on top of the CPR of the CT data set.

\subsection{D Cross Section}

By exploiting the vessel's center line, which has been computed as described in the previous subsection, we are also able to generate multimodal 2D cross section views of the vessel. We assume that we have a point of interest $p_{i}$, which is located on the center line (how to choose $p_{i}$ is described in Subsection 4.5). To visualize the cross section, we place a slice-shaped proxy geometry centered at $p_{i}$ and its normal pointing towards $t_{i}$. By deriving texture coordinates from $p_{i}$ and $t_{i}$, the desired information can be projected onto the slice. To display both modalities, PET and CT, we exploit multitexturing with an appropriate blending function. Therefore, we have to generate two texture coordinates for each vertex, one for each modality.

\subsection{Vessel Flattening}

To support comparative in-detail inspection of the wall thickness, the lumen diameter and the PET uptake at desired locations of different mouse models, we exploit a ray-based multimodal flattening approach. Flattening can be thought of as cutting one side of the vessel wall along the main axis and folding it open to reveal the interior. With our flattening approach, we aim at two application scenarios: (1) the in-detail exploration of individual aortic arches, and (2) the comparative visualization of different aortic arches to support a statistical analysis. While for the exploration scenario, spatial comprehension is important, it can be neglected when computing statistics. Therefore we have 


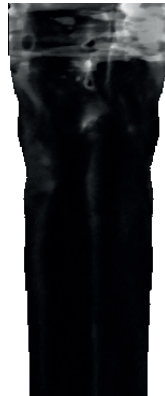

(a)

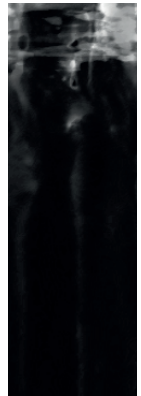

(b)

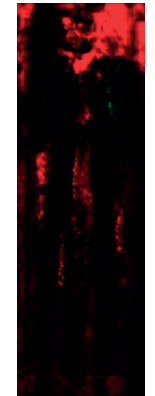

(c)

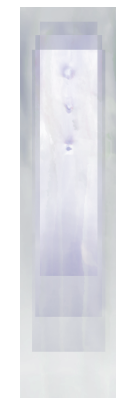

(d)

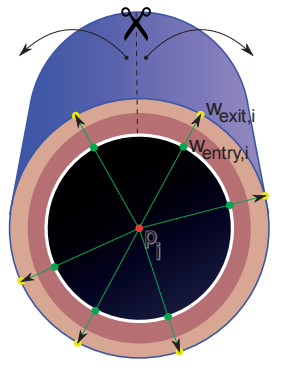

(a) normal case

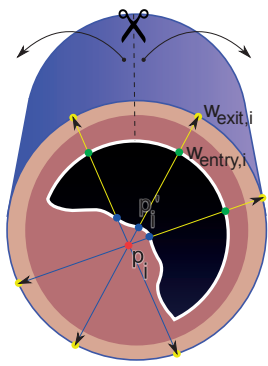

(b) $p_{i}$ inside plaque
Fig. 7. Variations of the described vessel flattening technique, which cuts and folds the vessel as illustrated in Figure 8. Wall thickness displayed with non-uniform thickness (a), and uniform thickness (b) as well as Z-score display (c). (d) shows the used control group encoded in RGBA. Since multiple data sets with different center line lengths contribute to the control group, it is displayed at a different scale. In (a) and (b) the luminance is proportional to the thickness, while (c) displays wall thickening in red and wall thinning in green.

realized two variations of the flattening approach. The first variant, the non-uniform width flattening, targets exploration and depicts besides the parameters described above also the diameter of the vessel (see Figure 7 (a)), while this information is not present in the second variant, the uniform width flattening (see Figure 7 (b)). As it can be seen, the non-uniform width flattening depicts the characteristic narrowing of the vessel diameter, while the uniform width flattening maps the vessel wall to a rectangle. Both variants are generated by exploiting ray-based vessel flattening, where rays are cast through a cross section of the vessel towards the vessel wall (see Figure 8 (a)). Casting these rays directly from the $p_{i}$ lying on the center line may lead to problems, since the $p_{i}$ can lie already inside the plaque, since we have computed the center line for the segment consisting of the lumen and the vessel wall (see Figure 8 (b)). Therefore for rays starting inside the plaque we have to determine a new starting position $p_{i}^{\prime}$. To achieve this, we distinguish between two cases when starting the ray casting inside the plaque. In the first case we do not encounter any lumen while marching outwards (blue rays). In this case we set $p_{i}^{\prime}$ to the first wall entry point in the opposite ray direction. In the second case, we do encounter lumen while marching outwards (yellow rays). In that case, we set $p_{i}^{\prime}$ to the first position outside the plaque. The lumen diameter is given by the length between the point on the center line $p_{i}$ and the first intersection with the vessel wall $w_{\text {entry, } i}$. Accordingly, the wall thickness is given by the length between $w_{\text {entry, } i}$ and the last intersection with the vessel wall $w_{\text {exit }, i}$.

While we cast a uniform number of rays for each cross section for the uniform width flattening, the number of rays varies depending on the vessel diameter when generating the non-uniform width flattening. To derive this number of rays for a specific $p_{i}$, we take into account the diameter of the whole vessel in the cross section at that position. When assuming that the section of the vessel lying in the cross cut can be approximated by a circle, we can set the numer of rays proportional to the number of voxels on the circle's circumference.

The drawback of ray-based flattening is that the application to vessels having a high degree of curvature may lead to crossing rays of adjacent cross sections, which would introduce artifacts. To reduce these artifacts, techniques have been presented which try to avoid intersection of rays of adjacent cross sections. For instance, Lee et al. propose the use of ray-templates for unfolding of virtual endoscopy [19]. Instead of casting rays, they use different ray cones depending on the curvature of the center line, and thus reduce the overlap of adjacent cross section planes. However, as it can be seen in the lower inset in Figure 6 (b), these ray intersections are not a problem in our case. As the circles in the inset depict, even in the regions of highest curvature of the aortic arches ray intersection points do not lie inside the vessel and thus do not lead to artifacts.
Fig. 8. Rays are cast to generate the flattening (a). Since the center line has been computed for a segment consisting of the lumen and the vessel wall, the $p_{i}$ might intersect the plaque and thus an adapted raycasting technique is required (b).

Both quantities, wall thickness and lumen diameter, are visualized using gray scale values. The idea behind this decision is based on the intensity depth cueing approach, which exploits that brighter regions are perceived closer compared to darker regions [29]. When looking at the flattened vessel wall, it can be considered as a height field, where thick wall regions are closer to the viewer than thin wall regions, and thick lumen diameters represent areas being further away. To derive the colors for the flattened PET map, we simply use a MIP compositing during the ray-casting and apply the chosen color lookup table.

To support a comparative statistical analysis of the uniform width flattening results, we compute and visualize the z-scores for lumen diameter and wall thickness. Therefore, we apply the ray-based flattening to the data sets belonging to the control group (Figure 7 (d)) and compute the mean value as well as the standard deviation. As seen in Figure 7, we can derive the z-scores for an individual data set and visualize them using a red-green color table. In the wall thickness display, we want to draw the users attention to areas where the wall is thickened, and therefore use red for positive $\mathrm{z}$-scores and green for negative $\mathrm{z}$-scores. In the lumen diameter display, we want to draw the attention to narrowed regions and have therefore chosen the inverse color mapping.

\subsection{Interaction Concepts}

The four different types of visualizations are linked together by means of an orientation icon, given by a gray line for the CPR and the flattening views and a disc for the $3 \mathrm{D}$ view. The position of this orientation icon indicates the position of the current point of interest $p_{i}$ and can be changed by using the scroll wheel on top of any of the four visualizations. Additionally, the user can click at any position on the vessel in the flattening displays to highlight this position as well as to display the most important quantitative results: wall thickness, lumen diameter and PET uptake. Since it has to be possible to inspect both modalities in the same space, we further provide a changeable PET/CT blending factor for the wall thickness flattening and the lumen diameter flattening in the non-comparative mode.

\section{Validation and Results}

Compared to regular single modality DVR, we introduce mainly two sources of uncertainty, first due to registration and then due to reformation. Therefore we have decided to validate the presented techniques based on two different phantoms. To validate the registration, we have scanned the phantom shown in Figure 9 (a)-(d). It consists of a disk-shaped object with differently sized compartments (drilled holes ranging from $0.5 \mathrm{~mm}$ to $2 \mathrm{~mm}$ ), which can be filled with contrast agent (Microfil) as well as PET tracer. To support the registration, we have added the same markers as used in the other preparations and scanned the phantom with PET and CT. The registration of the two modalities has been performed as described in Section 3. As Figure 9 (a)-(d) shows, the different structures are aligned in the two modalities after the registration. To validate the CPR as well as the flattening approach, 


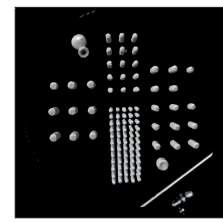

(a) $\alpha=0.00$

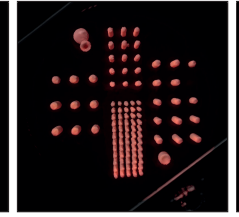

(b) $\alpha=0.50$

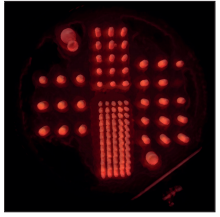

(c) $\alpha=0.75$

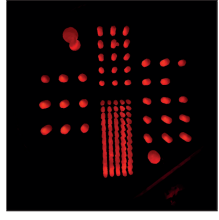

(d) $\alpha=1.00$

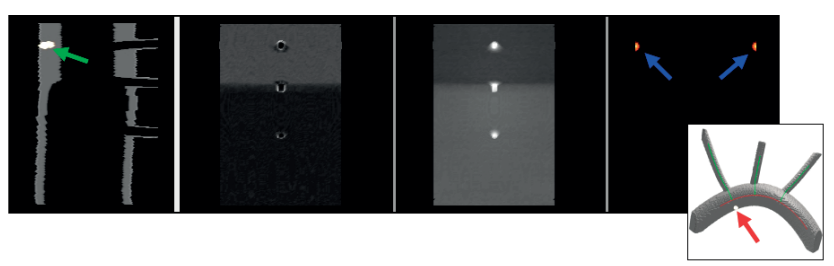

(e) multimodal phantom data set

Fig. 9. To validate the multimodal registration, we have equipped a phantom data set with registration markers. The images in (a-d) show a registered view of the PET and the CT scan, where the PET image is blended on top of the CT image using the specified $\alpha$ values. The visualization techniques are validated by exploiting a multimodal digital phantom data set, to which an artificial stenosis (red and green arrow) with a high PET uptake (blue arrows) has been added (e).

we have generated a digital phantom data set representing an idealized aortic arch with the three outgoing vessels. To validate the multimodal flattening as well as the localization of stenosis, we have integrated an artificial stenosis and generated a corresponding PET data set containing a spherical high uptake inside the stenosis. The visualization results of this multimodal phantom are shown in Figure 9 (e). As it can be seen, the stenosis (red arrow) is located properly in the CPR view (green arrow). Since the vessel is cut on the opposite side of the branches, the PET uptake is split and lies on both sides in the flattening view (blue arrows). Furthermore, the alignment of the CPR with the flattening display also matches, since the stenosis is on the same horizontal line as the PET uptake. Due to the binary nature of the phantom and the low resolution of $128^{3}$ voxels, sampling artifacts appear.

The presented visualization approach allows us for the first time to perform a quantitative and standardized comparison of groups of animals treated by different interventions. It enables us not only to look at an individual sample in a single mouse but also to assess differences in structure and molecular activity in an atherosclerotic mouse as compared to a group of control mice. We have exploited these benefits for intervention studies, where we have compared mice which have different genotypes and/or were fed with different diets to address questions, we could not answer before. In the following we exemplarily describe three cases, which we have evaluated during our studies. The first case shows results from a wildtype mouse (see Figure 10 (a), left CPR) as compared to an ApoE-deficient mouse under high cholesterol diet for 6 months (see Figure 10 (a), right CPR+flattenings). The visualization shows that the diet results in pronounced atherosclerosis in the aorta of the latter, while the left CPR shows normal wall thickness in the wild type mouse with no FDG uptake in the PET image. In contrast, the ApoE mouse shows clear signs of wall thickening in the inner curvature of the aortic arch which corresponds with a high uptake of F-18-FDG (radioactive glucose analogon) as marked by the white arrows. This can be interpreted as a typical finding of inflammation in the atherosclerotic vessel wall, where the accumulation of cholesterol together with immune cells (macrophages) increases wall thickness. Thus, macrophages can be imaged in different views, since they take up glucose (=FDG uptake) and do therefore result in the strong PET signal. This case enabled us to verify the presented visualization techniques in practice by evaluating these already well known facts. Currently we are going beyond known facts and exploit the system in order to image different stages of plaque development and assess the influence of different diets. Imaging of different plaque de-

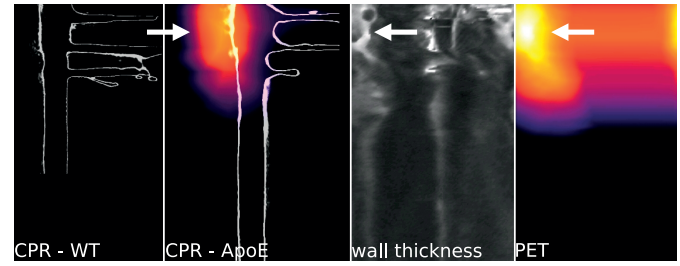

(a) wildtype mouse (left CPR) compared to ApoE-deficient mouse (F-18-FDG) (remaining views)

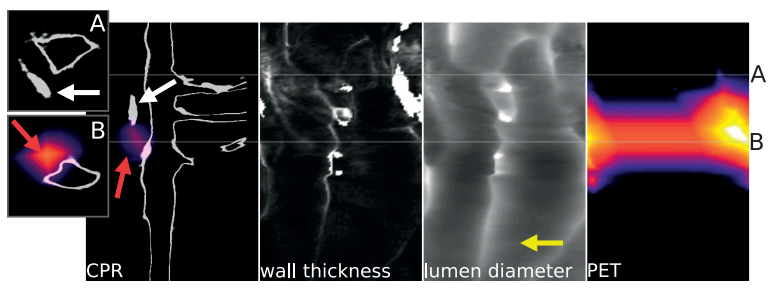

(b) ApoE-deficient mouse (F-18-CGS) fed with high cholesterol diet

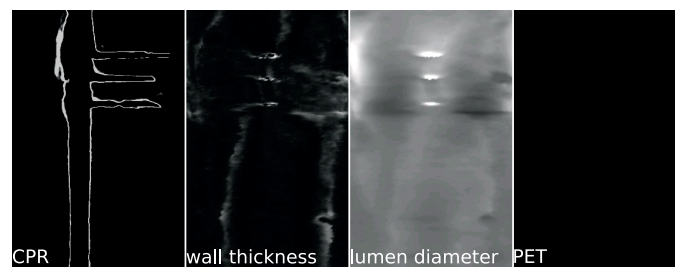

(c) ApoE-deficient mouse (F-18-CGS) fed regularly

Fig. 10. Application of the presented techniques to different mice, having a varying genetic background and/or diet.

velopment stages can be done since different PET markers allow to image different molecular processes in atherosclerosis. For instance, enzymes such as matrix-metalloproteinases (MMPs) are produced by the macrophages and destabilize atherosclerotic plaques. The following case describes our findings in this context when using F-18-CGS, a radioactively labeled enzyme inhibitor which is known to bind to atherosclerotic plaques [24]. While the behavior of F-18-FDG is very well understood, the behavior of F-18-CGS needs further investigation. In Figure 10 (b) we have injected F-18-CGS in a similar ApoE mouse as in the first case. Again, images show dramatic increases in wall thickness in the inner curvature and a inhomogeneity in lumen diameter indicating loss of the typical round shape of a vessel cross section, both characteristic signs of aortic atherosclerosis in CT. The ridges and valleys in the CT flattening views also depict the inhomogeneity in lumen diameter very well (yellow arrow). Calcium deposits (white arrow) can clearly be depicted from the CT scan in the atherosclerotic vessel wall. Most interestingly, in this case calcium spots (cross section A, white arrow) and enzyme activity (cross section B, red arrow) do not correlate. This observation supports the hypothesis that calcified areas in an atherosclerotic plaque are less inflamed whereas uncalcified ("soft") plaques can show a high degree of inflammation and thus soft plaques result in a high risk of future cardiovascular events, such as myocardial infarctions or stroke. While this hypothesis was under debate in the past, with our new visualization system we were able to identify cases supporting this hypothesis. The third case shows the strength of the proposed visualization concepts when looking for effects of intervention studies. To ensure that F-18CGS has a specific binding, we need to compare the previous case to an ApoE mouse fed without high cholesterol diet. Thus, in contrast to the ApoE mice in the first two examples, the ApoE mouse shown in Figure 10 (c) was not fed a high cholesterol diet which resulted in less pronounced atherosclerosis in the aorta. This is clearly visible in the CPR and the flattenings of the CT, also in contrast to the cholesterolfed mice the PET view does not show any inflammatory activity. The CT findings are also visible in the z-score display. We interpret this 
situation as a stable disease, where the risk of future cardiovascular events is rather low. Thus, we believe we found evidence for the specific binding of F-18-CGS in ApoE mice, and again the effect of the fed diet on the plaque development is shown nicely. To our knowledge, these necessary capabilities of detailed comparative visualization for different mice have not been established by any visualization approach so far. The application of the visualization concepts also confronted us with unsuspected findings, e.g., the non-correlation between changes in wall structure and molecular signal, which is under debate since some years. To get more insights, the presented visualization concepts will be used on a routine basis in larger cohorts of animal models of atherosclerosis involving in the future various known and new radiopharmaceuticals in order to better understand plaque development.

\section{Conclusion AND Future Work}

In this paper, we have presented a visualization system which allows to interactively explore PET/CT scans of mouse aortas in order to gain knowledge about the process of plaque development. We have presented new approaches to generate multipath CPR visualizations as well as to allow comparative visualization of different mice. By integrating these visualization techniques into a multi-view system, we were able to perform the analysis described in Section 5 by comparing individual mice against a control group. To validate the registration as well as the visualization techniques, we have used two different phantoms. In the future we would like to extend the system. By integrating the functionality to plan cuts through the data sets, the system can be used as a planning tool for subsequent histological examinations. Furthermore, we would like to exploit the computed wall thickness to perform a partial volume correction on the PET data.

\section{ACKNOWLEDGMENTS}

This work was partly supported by grants from the Deutsche Forschungsgemeinschaft (DFG), SFB 656 Cardiovascular Molecular Imaging Münster, Germany (projects A1, A3, C6, PM5, Z1, Z2), as well as the IZKF Münster, core unit SmAP and the EU FP7 Network of Excellence DiMI. The presented concepts have been integrated into the Voreen volume rendering engine (www.voreen.org).

\section{REFERENCES}

[1] J. Beyer, M. Hadwiger, S. Wolfsberger, and K. Bühler. High-quality multimodal volume rendering for preoperative planning of neurosurgical interventions. In IEEE Visualization, pages 1696-1703, 2007.

[2] R. Brecheisen, B. Platel, A. Vilanova, and B. ter Haar Romeny. Flexible GPU-based multi-volume ray-casting. In Vision, Modelling and Visualization, pages 1-6, 2008.

[3] T. Brunet, K. E. Nowak, and M. Gleicher. Integrating dynamic deformations into interactive volume visualization. In IEEE/EG EuroVis, pages 219-226, 2006.

[4] K. Bühler, P. Felkel, and A. L. Cruz. Geometric methods for vessel visualization and quantification- a survey. In Geometric Modelling for Scientific Visualization, pages 399-419. Springer, 2004.

[5] N. D. Cornea and P. Min. Curve-skeleton properties, applications, and algorithms. IEEE Trans. Visualization and Computer Graphics, 13(3):530548, 2007.

[6] N. D. Cornea, D. Silver, X. Yuan, and R. Balasubramanian. Computing hierarchical curve-skeletons of 3D objects. The Visual Computer, 21(11):945-955, 2005.

[7] A. Gerhards, P. Raab, S. Herber, K. F. Kreitner, T. Boskamp, and P. Mildenberger. Software-assisted CT-postprocessing of the carotid arteries. Rofo, 176(6):870-874, 2004.

[8] C. Glass and J. Witztum. Atherosclerosis. The road ahead. Cell, 104:503$516,2001$.

[9] H. K. Hahn, B. Preim, D. Selle, and H. O. Peitgen. Visualization and interaction techniques for the exploration of vascular structures. In IEEE Visualization, pages 395-402, 2001.

[10] S. He, R. Dai, B. Lu, C. Cao, H. Bai, and B. Jing. Medial axis reformation: a new visualization method for CT angiography. Acad Radiol, 8(8):726-733, 2001.

[11] W. Hong, X. Gu, F. Qiu, M. Jin, and A. Kaufman. Conformal virtual colon flattening. In ACM Solid and Physical Modeling, pages 85-93, 2006.
[12] R. Huang and K.-L. Ma. Rgvis: Region growing based techniques for volume visualization. In Pacific Conference on Computer Graphics and Applications, pages 355-363, 2003.

[13] A. Kanitsar, D. Fleischmann, R. Wegenkittl, P. Felkel, and M. E. Gröller. CPR: curved planar reformation. In IEEE Visualization, pages 37-44, 2002.

[14] A. Kanitsar, D. Fleischmann, R. Wegenkittl, and M. E. Gröller. Diagnostic relevant visualization of vascular structures. In Scientific Visualization: The Visual Extraction of Knowledge from Data, pages 207-228. Springer, 2006.

[15] A. Kanitsar, R. Wegenkittl, D. Fleischmann, and M. E. Gröller. Advanced curved planar reformation: Flattening of vascular structures. In IEEE Visualization, pages 43-50, 2003.

[16] P. Kohlmann, S. Bruckner, A. Kanitsar, and E. Gröller. Livesync: Deformed viewing spheres for knowledge-based navigation. In IEEE Visualization, pages 1544-1551, 2007.

[17] J. Krüger and R. Westermann. Acceleration techniques for GPU-based volume rendering. In IEEE Visualization, pages 287-292, 2003.

[18] D. H. Laidlaw. Geometric model extraction from magnetic resonance volume data. Technical report, 1995.

[19] H.-J. Lee, S. Lim, and B.-S. Shin. Unfolding of virtual endoscopy using ray-template. In Bio. and Medical Data Analysis, pages 69-77, 2005.

[20] N. Lee and M. Rasch. Tangential curved planar reformation for topological and orientation invariant visualization of vascular trees. In IEEE Engineering in Medicine and Biology Society, pages 1073-1076, 2006.

[21] S. Oeltze and B. Preim. Visualization of vasculature with convolution surfaces: method, validation and evaluation. IEEE Trans. Med. Imaging, 24(4):540-548, 2005.

[22] C. Rieder, F. Ritter, M. Raspe, and H.-O. Peitgen. Interactive visualization of multimodal volume data for neurosurgical tumor treatment. In IEEE/EG EuroVis, pages 1055-1062, 2008.

[23] R. Ross. Atherosclerosis-an inflammatory disease. N Engl J Med., 340:115-126, 1999

[24] M. Schäfers, B. Riemann, K. Kopka, H. Breyholz, S. Wagner, K. Schäfers, M. Law, O. Schober, and B. Levkau. Scintigraphic imaging of matrix metalloproteinase activity in the arterial wall in vivo. Circulation, 190(21):2554-2559, 2004.

[25] C. Schumann, S. Oeltze, R. Bade, B. Preim, and H.-O. Peitgen. Modelfree surface visualization of vascular trees. In IEEE/EG EuroVis, pages 283-290, 2007.

[26] R. R. Springmeyer, M. M. Blattner, and N. L. Max. A characterization of the scientific data analysis process. In IEEE Visualization, pages 235 242, 1992.

[27] M. Straka, M. Cervenanský, A. L. Cruz, A. Köchl, M. Srámek, M. E. Gröller, and D. Fleischmann. The vesselglyph: Focus \& context visualization in ct-angiography. In IEEE Visualization, pages 392-385, 2004.

[28] M. Tory. Mental registration of $2 \mathrm{~d}$ and $3 \mathrm{~d}$ visualizations (an empirical study). In IEEE Visualization, pages 371-378, 2003.

[29] D. Weiskopf and T. Ertl. Real-time depth-cueing beyond fogging. $J$. Graph. Tools, 7(4):83-90, 2002.

[30] D. Williams, S. Grimm, E. Coto, A. Roudsari, and H. Hatzakis. Volumetric curved planar reformation for virtual endoscopy. IEEE Trans. Visualization and Computer Graphics, 14(1):109-119, 2008.

[31] O. Wink, W. Niessen, and M. A. Viergever. Fast delineation and visualization of vessels in 3D angiographic images. IEEE Trans. Med. Imaging, 19(4):337-346, April 2000

[32] J. H. Won, G. D. Rubin, and S. Napel. Flattening the abdominal aortic tree for effective visualization. IEEE Engineering in Medicine and Biology Society, 1:3345-3348, 2006.

[33] Y. Yang, L. Zhu, S. Haker, A. Tannenbaum, and D. Giddens. Harmonic skeleton guided evaluation of stenoses in human coronary arteries. In Med. Image Comput. Comput. Assist. Interv., pages 490-497, 2005.

[34] L. Zhu, S. Haker, and A. Tannenbaum. Conformal flattening maps for the visualization of vessels. In SPIE Medical Imaging, volume 4681, pages 742-748, 2002.

[35] L. Zhu, S. Haker, and A. Tannenbaum. Flattening maps for the visualization of multibranched vessels. IEEE Trans. Med. Imaging, 24(2):191198, 2005.

[36] L. Zhu, S. Haker, A. Tannenbaum, S. Bouix, and K. Siddiqi. Anglepreserving mappings for the visualization of multi-branched vessels. In Image Processing 2002, volume 2, pages 945-948, 2002. 